\title{
Two dimensional kinetic analysis of electrostatic harmonic plasma waves
}

E. C. Fonseca-Pongutá, L. F. Ziebell, R. Gaelzer, and P. H. Yoon

Citation: Physics of Plasmas 23, 062310 (2016); doi: 10.1063/1.4953898

View online: http://dx.doi.org/10.1063/1.4953898

View Table of Contents: http://aip.scitation.org/toc/php/23/6

Published by the American Institute of Physics

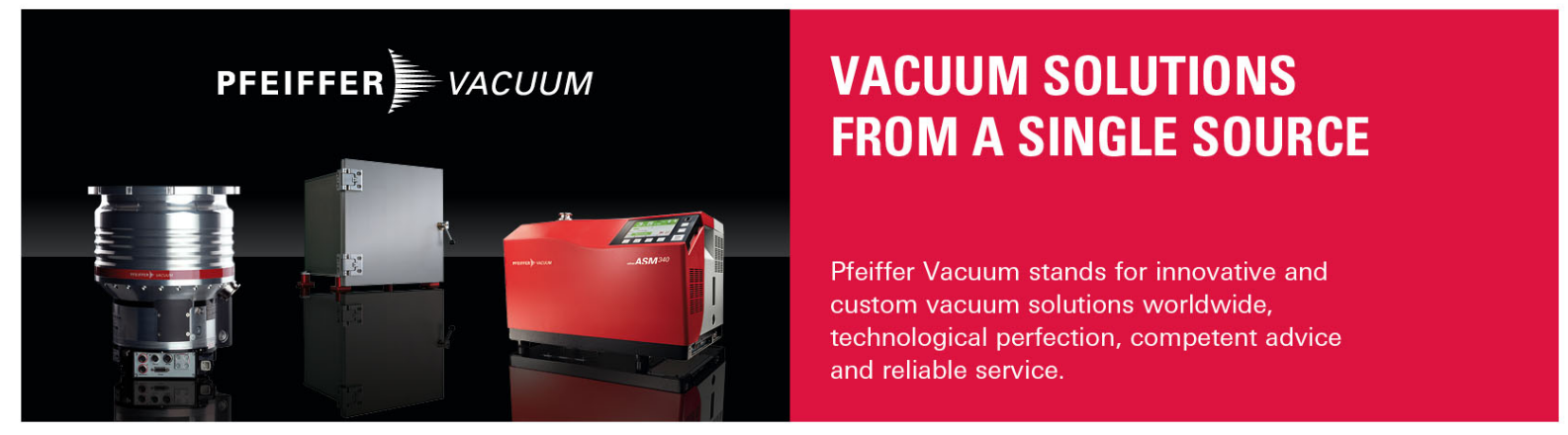




\title{
Two dimensional kinetic analysis of electrostatic harmonic plasma waves
}

\author{
E. C. Fonseca-Pongutá, ${ }^{1}$ L. F. Ziebell, ${ }^{1}$ R. Gaelzer, ${ }^{1}$ and P. H. Yoon ${ }^{2,3}$ \\ ${ }^{1}$ Instituto de Física, UFRGS, $91501-970$ Porto Alegre, RS, Brazil \\ ${ }^{2}$ IPST, University of Maryland, College Park, Maryland 20742, USA \\ ${ }^{3}$ SSR, Kyung Hee University, Yongin, Gyeonggi 446-701, South Korea
}

(Received 18 May 2016; accepted 31 May 2016; published online 17 June 2016)

Electrostatic harmonic Langmuir waves are virtual modes excited in weakly turbulent plasmas, first observed in early laboratory beam-plasma experiments as well as in rocket-borne active experiments in space. However, their unequivocal presence was confirmed through computer simulated experiments and subsequently theoretically explained. The peculiarity of harmonic Langmuir waves is that while their existence requires nonlinear response, their excitation mechanism and subsequent early time evolution are governed by essentially linear process. One of the unresolved theoretical issues regards the role of nonlinear wave-particle interaction process over longer evolution time period. Another outstanding issue is that existing theories for these modes are limited to one-dimensional space. The present paper carries out two dimensional theoretical analysis of fundamental and (first) harmonic Langmuir waves for the first time. The result shows that harmonic Langmuir wave is essentially governed by (quasi)linear process and that nonlinear wave-particle interaction plays no significant role in the time evolution of the wave spectrum. The numerical solutions of the two-dimensional wave spectra for fundamental and harmonic Langmuir waves are also found to be consistent with those obtained by direct particle-in-cell simulation method reported in the literature. Published by AIP Publishing.

[http://dx.doi.org/10.1063/1.4953898]

\section{INTRODUCTION}

In collisionless kinetic theory, plasmas are described by Maxwell equations for the electric and magnetic fields and Vlasov equations for the distribution functions of different species of particles contained in the plasma. The Vlasov-Maxwell system of equations is a complex system, frequently discussed in the context of approximations, such as the linear and quasilinear approximations. Under the linear approximation, one obtains dispersion relations which lead to the identification of the modes of oscillation that are excited and propagate in the plasma system. For instance, the linear dispersion relations lead to the identification of high frequency electrostatic waves, associated with electron oscillations, which are known as Langmuir waves $(L)$, and also to the identification of lower frequency electrostatic oscillations in which both ions and electrons participate, which are known as ion-acoustic waves $(S)$. For magnetized plasmas, the linear dispersion relations also lead to a bewildering array of small-amplitude oscillations, one of the most well-known example being the lowfrequency electromagnetic oscillations, known as Alfvén wave, for instance.

In addition to information regarding the relationship between wave number and real frequency, the linear dispersion relations also contain information on the imaginary parts of the wave frequencies, which describe damping or growth of waves due to resonance between waves and particles. However, the linear approximation does not have dynamical information concerning the time evolution, for which one must go beyond linear theory, the lowest order theory being the quasilinear theory. The next-order theory is known as the weak turbulence theory (WT), developed in the time period between late 1950s to early 1970 s, in which important contributions were made by scientists of the former Soviet Union. ${ }^{1-9}$ More recently, the formalism of the WT theory was revisited ab initio, ${ }^{10}$ initially without considering the effects of single-particle fluctuations, but later by incorporating these effects. ${ }^{11}$ The formalism was subsequently extended to include the effects of electromagnetic oscillations. ${ }^{12,13}$ More recently, the electrostatic part of the renewed formalism was further extended to incorporate effects due to binary interactions of particles, leading to a collisional term in the equations for the particle distributions, and to new terms in the equations for the time evolution of wave amplitudes, identified with the physical processes of collisional damping, spontaneous emission of electrostatic waves by binary particle interactions, and effects due to binary particle interactions in the processes of wave scattering. ${ }^{14,15}$

The reformulation of the WT theory, which was presented in Ref. 10 included the presence of electrostatic waves with frequency close to $2 \omega_{p e}$, where $\omega_{p e}=\left(4 \pi \hat{n} e^{2} / m_{e}\right)^{1 / 2}$ is the plasma frequency of electrons, $\hat{n}$ being the ambient plasma density, and $e$ and $m_{e}$ being the unit electric charge and electron mass, respectively. These waves were identified as the harmonic of $L$ waves, and the formalism led to a set of coupled equations for description of the evolution of $L$ waves, fundamental and harmonic, $S$ waves, and particles. ${ }^{10}$ The $S$ waves and $L$ wave at the fundamental frequency are normal modes of the plasma, predicted by linear dispersion relations, but the harmonic of $L$ wave is not a solution to the linear dispersion relation, but rather, its presence can be described only if one includes nonlinear correction to the linear dispersion relation. In subsequent papers, higher order electrostatic 
harmonics were also discussed. ${ }^{11,16-18}$ These theoretical developments provided support to the recognized occurrence of $n \omega_{p e}$ electrostatic perturbations as virtual modes excited in weakly turbulent plasmas.

The excitations of such modes were first detected in early laboratory beam-plasma experiments, ${ }^{19-23}$ and also in space-borne rocket active experiment. ${ }^{24}$ However, the above-referenced early works did not realize the peculiarity of harmonic electrostatic modes. Instead, interpretations tended to regard these excitations as related to customary nonlinear phenomena such as wave trapping. The strange behavior associated with the harmonic $L$ modes did not fully exhibit itself until the phenomenon was observed in computer simulated experiments. ${ }^{25-31}$ Unlike typical nonlinear phenomena, which do not manifest themselves unless the wave amplitude that drives these phenomena exceeds certain threshold, the electrostatic harmonics are excited very early on during the beam-plasma instability process. In fact, the growth rate of the $n$-th harmonic mode is higher than the fundamental $L$ mode growth rate by roughly the multiple of $n$. Subsequent time development and saturation are dictated by essentially (quasi) linear theory. These behaviors are well described by the theory that interpret these modes as solutions to nonlinear dispersion relation, but their dynamics as being governed by quasilinear theory. ${ }^{16-18}$

Space observations show that multiple harmonic electron plasma frequency fluctuations are often seen in the Earth's collisionless bow shock environment and even in interplanetary type III radio burst source region. ${ }^{32-36}$ While alternative interpretations for the occurrence of these emissions are possible, more recent simulation studies confirm the ubiquitous excitations of harmonic $L$ mode during beamplasma instability process. $^{37-39}$

A hitherto unanswered question concerning the possible occurrence of both electrostatic and electromagnetic harmonics was addressed in another paper, ${ }^{40}$ which lead to the conclusion that both modes are independent solutions. ${ }^{40}$ Indeed, in related works in which two-dimensional (2D) electromagnetic particle-in-cell simulation code was employed, Refs. 30 and 43 demonstrate that both electric and magnetic field fluctuations are characterized by multiple harmonic spectra.

To the best of our knowledge, numerical analysis of the generalized WT theory including the effect of electrostatic harmonics have been made only in the context of one-dimensional analysis (1D). The first published application that made use of the formalism presented in Ref. 10 appeared in the year 2002, with the discussion of the time evolution of beam-plasma instability, taking into account fundamental and harmonics $L$ waves and $S$ waves, and by incorporating quasilinear effect of induced emission and the nonlinear effects of three-wave decay and scattering. ${ }^{41}$ Reference 41 did not take into account spontaneous effects, included in the general formalism after Ref. 11, but only collective nonlinear effects. Shortly after Ref. 11, discreteparticle effects were incorporated in Ref. 42, but the analysis still pertained to $1 \mathrm{D}$ approximation.

On the other hand, a two-dimensional (2D) formulation has been used by some of us for the analysis of different aspects of the beam-plasma interaction, but always focusing on the time evolution of the particle distribution functions and of the amplitudes of normal modes predicted by linear theory. We have started these 2D analysis in the year 2008, discussing the evolution of the electrostatic modes, ${ }^{44,45}$ and have continued with the subject along subsequent years. In some of the more recent works, we have also considered electromagnetic waves into the WT analysis, and discussed the emission of electromagnetic waves by nonlinear processes, considering the paradigmatic case of the plasma emission, ${ }^{46,47}$ and also the possibility of emission without the presence of a beam. ${ }^{48,49}$

The 2D analysis provides information and insights that are not immediately available with $1 \mathrm{D}$ analysis. There are certain features such as the time scales of different nonlinear processes, which may be adequately addressed by $1 \mathrm{D}$ analysis; other features such as the angular distribution of the radiation by plasma emission, for instance, can be learned only through a 2D formulation. Another example may be the isotropization of $L$ waves in 2D $k$ space, which could not have been predicted by $1 \mathrm{D}$ analysis. ${ }^{44,45,50}$

The purpose of the present paper is to perform 2D analysis of the beam-plasma instability process including the nonlinear eigenmode, i.e., the first harmonic $L$ mode, or $2 \omega_{p e}$ electrostatic mode, for the first time. The present investigation aims to address a specific issue related to the nonlinear $2 \omega_{p e}$ electrostatic mode excitation, but the general 2D solution is useful in and of itself. For instance, one may compare the present theory with some simulation results already available in the literature. Specifically, Ref. 43 contains Figures 3(b) and 3(c) that show the 2D spectrum of fundamental and second harmonic electric field fluctuation. Similar result is also found in Ref. 30. The present 2D calculation can be directly compared against such results. The specific issue that the present paper aims to address concerns a theoretical conjecture presented in Ref. 11, where it was speculated that the early dynamical evolution of $2 \omega_{p e}$ mode should follow the dictates of quasilinear theory, but for later times, nonlinear wave-particle interaction process, that is, induced scattering, should dominate. The present analysis seeks to test this hypothesis.

In the present paper we present and briefly discuss the basic equations of WT theory, which will be employed in order to describe the time evolution of the particle distributions and electrostatic modes, including the harmonics of the $L$ waves, and obtain the $2 \mathrm{D}$ versions of these equations. These 2D equations will therefore be utilized for numerical analysis of the beam-plasma instability. For simplicity of this first analysis, which includes the effect of $L$ harmonics in $2 \mathrm{D}$, we restrict ourselves to a formulation that does not consider electromagnetic effects, and also neglect the effects of harmonics higher than $n=2$.

The structure of the paper is as follows: In Sec. II we briefly describe the theoretical formulation and the setup for the numerical analysis. Section III presents the results of numerical analysis. We summarize our findings in Sec. IV.

\section{THEORETICAL FORMULATION AND NUMERICAL SETUP}

For the purpose of the present paper, we start from a general self-consistent set of equations that include only the 
effect of electrostatic waves, as they have appeared in Ref. 13. We present here these equations using non-dimensional variables, which are more suitable for numerical analysis

$$
z \equiv \frac{\omega}{\omega_{p e}}, \quad \tau \equiv t \omega_{p e}, \quad \mathbf{q} \equiv \frac{\mathbf{k} v_{t e}}{\omega_{p e}}, \quad \mathbf{u} \equiv \frac{\mathbf{v}}{v_{t e}},
$$

where $v_{t e}=\left(2 T_{e} / m_{e}\right)^{1 / 2}$ is electron thermal speed, $T_{e}$ being the temperature defined in energy unit, and we also utilize normalized distribution functions and wave spectra

$$
\Phi_{a}(\mathbf{u})=v_{t e}^{3} F_{a}(\mathbf{v}), \quad \mathcal{E}_{\mathbf{q}}^{\sigma \alpha}=\frac{(2 \pi)^{2} g}{m_{e} v_{t e}^{2}} \frac{I_{\mathbf{k}}^{\sigma \alpha}}{\mu_{\mathbf{k}}^{\alpha}} .
$$

Moreover, we define some useful quantities

$$
\begin{gathered}
\mu_{\mathbf{q}}^{L}=1, \quad \mu_{\mathbf{q}}^{S}=\frac{q^{3}}{2^{3 / 2}} \sqrt{\frac{m_{e}}{m_{i}}}\left(1+\frac{3 T_{i}}{T_{e}}\right)^{1 / 2}, \\
\lambda_{D e}^{2}=\frac{T_{e}}{4 \pi \hat{n} e^{2}}=\frac{v_{t e}^{2}}{2 \omega_{p e}^{2}}, \quad g=\frac{1}{2^{3 / 2}(4 \pi)^{2} \hat{n} \lambda_{D e}^{3}} .
\end{gathered}
$$

In the above $\lambda_{D e}=\left[T_{e} /\left(4 \pi \hat{n} e^{2}\right)\right]^{1 / 2}$ is the Debye length such that $1 /\left(\hat{n} \lambda_{D e}^{3}\right)$ represents the plasma parameter.

The equation for the time evolution of the fundamental $L$ wave can be written in dimensionless form as follows:

$$
\begin{aligned}
\frac{\partial \mathcal{E}_{\mathbf{q}}^{\sigma L}}{\partial \tau}= & \left\{\mu_{\mathbf{q}}^{L} \frac{\pi}{q^{2}} \int d \mathbf{u} \delta\left(\sigma z_{\mathbf{q}}^{L}-\mathbf{q} \cdot \mathbf{u}\right)\left(g \Phi_{e}(\mathbf{u})+\left(\sigma z_{\mathbf{q}}^{L}\right) \mathbf{q} \cdot \frac{\partial \Phi_{e}(\mathbf{u})}{\partial \mathbf{u}} \mathcal{E}_{\mathbf{q}}^{\sigma L}\right)\right\}_{L q l} \\
& +\left\{2 \sigma \mu_{\mathbf{q}}^{L} z_{\mathbf{q}}^{L} \sum_{\sigma^{\prime}, \sigma^{\prime \prime}= \pm 1} \int d \mathbf{q}^{\prime} \frac{\mu_{\mathbf{q}^{\prime}}^{L} \mu_{\mathbf{q}-\mathbf{q}^{\prime}}^{S}\left(\mathbf{q} \cdot \mathbf{q}^{\prime}\right)^{2}}{q^{2} q^{\prime 2}\left|\mathbf{q}-\mathbf{q}^{\prime}\right|^{2}}\left[\sigma z_{\mathbf{q}}^{L} \mathcal{E}_{\mathbf{q}^{\prime}}^{\sigma^{\prime} L} \mathcal{E}_{\mathbf{q}^{\sigma^{\prime \prime} \mathbf{q}^{\prime}}}^{\sigma^{\prime \prime}}-\left(\sigma^{\prime} z_{\mathbf{q}^{\prime}}^{L} \mathcal{E}_{\mathbf{q}-\mathbf{q}^{\prime}}^{\sigma^{\prime \prime} S}+\sigma^{\prime \prime} z_{\mathbf{q}-\mathbf{q}^{\prime}}^{L} \mathcal{E}_{\mathbf{q}^{\prime}}^{\sigma^{\prime} L}\right) \mathcal{E}_{\mathbf{q}}^{\sigma L}\right]\right. \\
& \left.\times \delta\left(\sigma z_{\mathbf{q}}^{L}-\sigma^{\prime} z_{\mathbf{q}^{\prime}}^{L}-\sigma^{\prime \prime} z_{\mathbf{q}-\mathbf{q}^{\prime}}^{S}\right)\right\}_{L d L S}+\left\{\sigma z_{\mathbf{q}}^{L} \sum_{\sigma^{\prime}} \int d \mathbf{q}^{\prime} \int d \mathbf{u} \frac{\mu_{\mathbf{q}}^{L} \mu_{\mathbf{q}^{\prime}}^{L}\left(\mathbf{q} \cdot \mathbf{q}^{\prime}\right)^{2}}{q^{2} q^{\prime 2}\left[\sigma z_{\mathbf{q}}^{L}-\sigma^{\prime} z_{\mathbf{q}^{\prime}}^{L}-\left(\mathbf{q}-\mathbf{q}^{\prime}\right) \cdot \mathbf{u}\right]}\right. \\
& \left.\times\left[g\left(\sigma z_{\mathbf{q}}^{L} \mathcal{E}_{\mathbf{q}^{\prime}}^{\sigma^{\prime} L}-\sigma^{\prime} z_{\mathbf{q}^{\prime}}^{L} \mathcal{E}_{\mathbf{q}}^{\sigma L}\right)\left[\Phi_{e}(\mathbf{u})+\Phi_{i}(\mathbf{u})\right]+\frac{m_{e}}{m_{i}} \mathcal{E}_{\mathbf{q}^{\prime}}^{\sigma^{\prime} L} \mathcal{E}_{\mathbf{q}}^{\sigma L}\left(\mathbf{q}-\mathbf{q}^{\prime}\right) \cdot \frac{\partial \Phi_{i}(\mathbf{u})}{\partial \mathbf{u}}\right]\right\}_{L s L} .
\end{aligned}
$$

The terms appearing in Eq. (1) can be described as follows. The first term on the right-hand side, enclosed within the large curly brackets, is denoted by subscript Lql. It describes the spontaneous emission and quasilinear (i.e., induced emission) effects for the $L$ mode. The second term describes the effects of three-wave decay involving $L$ and $S$ mode waves, and is denoted as $L d L S$. The third term stands for the scattering process involving $L$ waves, and its designation is $L s L L$.

For $S$ mode, the normalized dynamical equation is the following:

$$
\begin{aligned}
\frac{\partial \mathcal{E}_{\mathbf{q}}^{\sigma S}}{\partial \tau}= & \left\{\mu_{\mathbf{q}}^{S} \frac{\pi}{q^{2}} \int d \mathbf{u} \delta\left(\sigma z_{\mathbf{q}}^{S}-\mathbf{q} \cdot \mathbf{u}\right)\left[g\left[\Phi_{e}(\mathbf{u})+\Phi_{i}(\mathbf{u})\right]+\left(\sigma z_{\mathbf{q}}^{L}\right)\left(\mathbf{q} \cdot \frac{\partial \Phi_{e}(\mathbf{u})}{\partial \mathbf{u}}+\frac{m_{e}}{m_{i}} \mathbf{q} \cdot \frac{\partial \Phi_{i}(\mathbf{u})}{\partial \mathbf{u}}\right) \mathcal{E}_{\mathbf{q}}^{\sigma S}\right]\right\}_{S q l} \\
& +\left\{\sigma z_{\mathbf{q}}^{L} \sum_{\sigma^{\prime}, \sigma^{\prime \prime}} \int d \mathbf{q}^{\prime} \frac{\mu_{\mathbf{q}}^{S} \mu_{\mathbf{q}^{\prime}}^{L} \mu_{\mathbf{q}-\mathbf{q}^{\prime}}^{L}\left[\mathbf{q}^{\prime} \cdot\left(\mathbf{q}-\mathbf{q}^{\prime}\right)\right]^{2}}{q^{2} q^{\prime 2}\left|\mathbf{q}-\mathbf{q}^{\prime}\right|^{2}}\left[\sigma z_{\mathbf{q}}^{L} \mathcal{E}_{\mathbf{q}^{\prime}}^{\sigma^{\prime} L} \mathcal{E}_{\mathbf{q}-\mathbf{q}^{\prime}}^{\sigma^{\prime \prime} L}-\left(\sigma^{\prime} z_{\mathbf{q}^{\prime}}^{L} \mathcal{E}_{\mathbf{q}-\mathbf{q}^{\prime}}^{\sigma^{\prime \prime} L}+\sigma^{\prime \prime} z_{\mathbf{q}-\mathbf{q}^{\prime}}^{L} \mathcal{E}_{\mathbf{q}^{\prime}}^{\sigma^{\prime} L}\right) \mathcal{E}_{\mathbf{q}}^{\sigma S}\right]\right. \\
& \left.\times \delta\left(\sigma z_{\mathbf{q}}^{S}-\sigma^{\prime} z_{\mathbf{q}^{\prime}}^{L}-\sigma^{\prime \prime} z_{\mathbf{q}-\mathbf{q}^{\prime}}^{L}\right)\right\}_{S d L L} .
\end{aligned}
$$

The first term at the right-hand side of Eq. (2) describes the spontaneous emission and quasilinear effects, and is denoted as Sql. The second term describes the three-wave decay process and is designated by $S d L L$.

For the harmonics of $L$ waves, the time evolution equation is as follows: ${ }^{11,42}$

$$
\frac{\partial \mathcal{E}_{\mathbf{q}}^{L n}}{\partial \tau}=\frac{\gamma_{\mathbf{q}}^{L n}+\nu_{\mathbf{q}}^{L n}}{1+\eta_{\mathbf{q}}^{L n}} \mathcal{E}_{\mathbf{q}}^{L n}
$$

where

$$
\gamma_{\mathbf{q}}^{L n}=n^{2} \frac{\pi}{q^{2}} \int d \mathbf{u} z_{\mathbf{q}}^{L n} \mathbf{q} \cdot \frac{\partial \Phi(\mathbf{u})}{\partial \mathbf{u}} \delta\left(\sigma z_{\mathbf{q}}^{L n}-\mathbf{q} \cdot \mathbf{u}\right),
$$




$$
\begin{aligned}
\nu_{\mathbf{q}}^{L n}= & n^{3} \int d \mathbf{q}^{\prime} \frac{a_{\mathbf{q}, \mathbf{q}^{\prime}}^{n} \mu_{\mathbf{q}^{\prime}}^{L(n-1)}\left(z_{\mathbf{q}}^{L n}-z_{\mathbf{q}^{\prime}}^{L(n-1)}\right)}{\left|\epsilon\left(\mathbf{q}-\mathbf{q}^{\prime}, z_{\mathbf{q}}^{L n}-z_{\mathbf{q}^{\prime}}^{L(n-1)}\right)\right|^{2}} \\
& \times \mathcal{E}_{\mathbf{q}^{\prime}}^{L(n-1)} \int d \mathbf{u} \frac{\mathbf{q}-\mathbf{q}^{\prime}}{\left|\mathbf{q}-\mathbf{q}^{\prime}\right|^{2}} \cdot \frac{\partial \Phi_{e}}{\partial \mathbf{u}} \\
& \times \delta\left[z_{\mathbf{q}}^{L n}-z_{\mathbf{q}^{\prime}}^{L(n-1)}-\left(\mathbf{q}-\mathbf{q}^{\prime}\right) \cdot \mathbf{u}\right], \\
\eta_{\mathbf{q}}^{L n}= & \frac{n^{3}}{\pi} \int d \mathbf{q}^{\prime} a_{\mathbf{q}, \mathbf{q}^{\prime}}^{n} \mu_{\mathbf{q}}^{L(n-1)} \mathcal{E}_{\mathbf{q}^{\prime}}^{L(n-1)} \\
& \times \operatorname{Re}\left[\epsilon^{-2}\left(\mathbf{q}-\mathbf{q}^{\prime}, z_{\mathbf{q}}^{L n}-z_{\mathbf{q}^{\prime}}^{L(n-1)}\right)\right] .
\end{aligned}
$$

The equation for the evolution of nonlinear modes, Eq. (3), contains terms associated to quasilinear (induced emission) and to nonlinear effects. The term with $\gamma_{\mathbf{q}}^{L n}$ represents the quasilinear effect, and the term with $\nu_{\mathbf{q}}^{L n}$ represents the effect of wave-particle scattering. ${ }^{11,42}$

For the evaluation of various objects defined in Eqs. (5) and (6), we utilize

$$
\begin{aligned}
a_{\mathbf{q}, \mathbf{q}^{\prime}}^{n}= & \left\{(n-1) q^{2}\left[\mathbf{q}^{\prime} \cdot\left(\mathbf{q}-\mathbf{q}^{\prime}\right)\right]+n q^{\prime 2}\left[\mathbf{q} \cdot\left(\mathbf{q}-\mathbf{q}^{\prime}\right)\right]\right. \\
+ & \left.n(n-1)\left|\mathbf{q}-\mathbf{q}^{\prime}\right|^{2}\left(\mathbf{q} \cdot \mathbf{q}^{\prime}\right)\right\}^{2} \\
& \times\left[n^{2}(n-1)^{2} q q^{\prime}\left|\mathbf{q}-\mathbf{q}^{\prime}\right|\right]^{-2}, \\
\left|\epsilon\left(\mathbf{q}-\mathbf{q}^{\prime}, z_{\mathbf{q}}^{L n}-z_{\mathbf{q}^{\prime}}^{L(n-1)}\right)\right|^{2}= & 4\left[\left(z_{\mathbf{q}}^{L n}-z_{\mathbf{q}^{\prime}}^{L(n-1)}-z_{\mathbf{q}-\mathbf{q}^{\prime}}^{L 1}\right)^{2}\right. \\
& \left.+\pi \xi_{\mathbf{q}-\mathbf{q}^{\prime}}^{6} e^{-2 \xi_{\mathbf{q}-\mathbf{q}^{\prime}}^{2}}\right],
\end{aligned}
$$

$\operatorname{Re} \epsilon^{-2}\left(\mathbf{q}-\mathbf{q}^{\prime}, z_{\mathbf{q}}^{L n}-z_{\mathbf{q}^{\prime}}^{L(n-1)}\right)=\frac{\left(z_{\mathbf{q}}^{L n}-z_{\mathbf{q}^{\prime}}^{L(n-1)}-z_{\mathbf{q}-\mathbf{q}^{\prime}}^{L 1}\right)^{2}}{\left|\epsilon\left(\mathbf{q}-\mathbf{q}^{\prime}, z_{\mathbf{q}}^{L n}-z_{\mathbf{q}^{\prime}}^{L(n-1)}\right)\right|^{2}}$,

with

$$
\xi_{\mathbf{q}-\mathbf{q}^{\prime}}=\frac{z_{\mathbf{q}-\mathbf{q}^{\prime}}^{L 1}}{\left|\mathbf{q}-\mathbf{q}^{\prime}\right|}
$$

In the limit $\left|\xi_{\mathbf{q}, \mathbf{q}^{\prime}}\right| \gg 1$, Eqs. (8) and (9) are given, respectively, by

$$
\begin{gathered}
\left|\epsilon\left(\mathbf{q}-\mathbf{q}^{\prime}, z_{\mathbf{q}}^{L n}-z_{\mathbf{q}^{\prime}}^{L(n-1)}\right)\right|^{2} \simeq 4\left(z_{\mathbf{q}}^{L n}-z_{\mathbf{q}^{\prime}}^{L(n-1)}-z_{\mathbf{q}-\mathbf{q}^{\prime}}^{L 1}\right)^{2}, \quad(10) \\
\operatorname{Re} \epsilon^{-2}\left(\mathbf{q}-\mathbf{q}^{\prime}, z_{\mathbf{q}}^{L n}-z_{\mathbf{q}^{\prime}}^{L(n-1)}\right) \simeq \frac{1}{4\left(z_{\mathbf{q}}^{L n}-z_{\mathbf{q}^{\prime}}^{L(n-1)}-z_{\mathbf{q}-\mathbf{q}^{\prime}}^{L 1}\right)^{2}},
\end{gathered}
$$

and therefore Eqs. (5) and (6) are given by the approximated expressions that appear as Equation (33) in Ref. 11 and Equation (7) in Ref. 42.

The set of equations which has been obtained for the amplitudes of electrostatics waves in an unmagnetized plasma, comprising $L, S$, and harmonic modes, must be solved along with the dynamical equations for the particle distribution functions

$$
\begin{aligned}
\frac{\partial \Phi_{a}(\mathbf{u})}{\partial \tau}= & \frac{e_{a}^{2}}{e^{2}} \frac{m_{e}^{2}}{m_{a}^{2}} \sum_{\sigma} \sum_{\alpha=L, S} \int d \mathbf{q}\left(\frac{\mathbf{q}}{q} \cdot \frac{\partial}{\partial \mathbf{u}}\right) \mu_{\mathbf{q}}^{\alpha} \delta\left(\sigma z_{\mathbf{q}}^{\alpha}-\mathbf{q} \cdot \mathbf{u}\right) \\
& \times\left(g \frac{m_{a}}{m_{e}} \frac{\sigma z_{\mathbf{q}}^{L}}{q} \Phi_{a}(\mathbf{u})+\mathcal{E}_{\mathbf{q}}^{\sigma \alpha} \frac{\mathbf{q}}{q} \cdot \frac{\partial \Phi_{a}(\mathbf{u})}{\partial \mathbf{u}}\right) .
\end{aligned}
$$

In the above, $a=e$ denote the electrons and $a=i$ stands for the ions. The dispersion relations for plasma normal modes $L$ and $S$ in terms of non-dimensional variables are given by the following expressions:

$$
\begin{aligned}
z_{\mathbf{q}}^{L} & =\left(1+\frac{3}{2} q^{2}\right)^{1 / 2} \\
z_{\mathbf{q}}^{S} & =\frac{q A}{\left(1+q^{2} / 2\right)^{1 / 2}},
\end{aligned}
$$

where

$$
A=\frac{1}{\sqrt{2}}\left(\frac{m_{e}}{m_{i}}\right)^{1 / 2}\left(1+\frac{3 T_{i}}{T_{e}}\right)^{1 / 2} .
$$

For the harmonic waves, the dispersion relation may be written as follows: ${ }^{11,16}$

$$
z_{\mathbf{q}}^{L n}=\left(n+\varepsilon_{\mathbf{q}}^{n}+\frac{3}{4} q^{2}+\frac{3 \theta_{\mathbf{q}}^{n}}{\varepsilon_{\mathbf{q}}^{n}} \lambda_{D e}^{2}\right),
$$

where

$$
\varepsilon_{\mathbf{q}}^{n}=\frac{1}{8 \pi} \frac{n^{2}}{(n-1)} \int d^{3} \mathbf{q}^{\prime} a_{\mathbf{q}, \mathbf{q}^{\prime}}^{n} \mu_{\mathbf{q}^{\prime}}^{L(n-1)} \mathcal{E}_{\mathbf{q}^{\prime}}^{L(n-1)} .
$$

Taking into account that $\mu_{\mathbf{q}}^{L n}=1$ for $n=1,2, \ldots$, and that $\varepsilon_{\mathbf{q}}^{1}=0$, the following form can be obtained:

$$
\varepsilon_{\mathbf{q}}^{n}=\frac{1}{8 \pi} \frac{n^{2}}{(n-1)} \int d^{3} \mathbf{q}^{\prime} a_{\mathbf{q}, \mathbf{q}^{\prime}}^{n} \mathcal{E}_{\mathbf{q}^{\prime}}^{L(n-1)},
$$

with the function $\theta_{\mathbf{q}}^{n}$ given in the following form:

$$
\begin{aligned}
\lambda_{D e}^{2} \theta_{\mathbf{q}}^{n}= & \frac{1}{8 \pi} \frac{n^{2}}{(n-1)} \int d^{3} \mathbf{q}^{\prime} a_{\mathbf{q}, \mathbf{q}^{\prime}}^{n} \mathcal{E}_{\mathbf{q}^{\prime}}^{L(n-1)} \\
& \times\left(\frac{q^{\prime 2}-\mathbf{q} \cdot \mathbf{q}^{\prime}}{2}+\frac{\theta_{\mathbf{k}^{\prime}}^{n-1} \lambda_{D e}^{2}}{\varepsilon_{\mathbf{k}^{\prime}}^{n-1}}\right) .
\end{aligned}
$$

At this point, some qualitative comments can be made about the time evolution of harmonic modes. For the initial stages of the time evolution, when the wave intensity satisfies the condition $\mathcal{E}_{\mathbf{q}^{\prime}}^{L(n-1)} \ll\left(z_{\mathbf{q}}^{L n}-z_{\mathbf{q}^{\prime}}^{L(n-1)}-z_{\mathbf{q}-\mathbf{q}^{\prime}}^{L 1}\right)^{2}$, the linear part of kinetic equation (6) must be dominant ${ }^{11}$

$$
\frac{\partial \mathcal{E}_{\mathbf{q}}^{L n}}{\partial \tau} \sim \gamma_{\mathbf{q}}^{L n} \mathcal{E}_{\mathbf{q}}^{L n} .
$$

For continued evolution, it was predicted in Ref. 11 that the system would attain $\mathcal{E}_{\mathbf{q}^{\prime}}^{L(n-1)} \gg\left(z_{\mathbf{q}}^{L n}-z_{\mathbf{q}^{\prime}}^{L(n-1)}-z_{\mathbf{q}-\mathbf{q}^{\prime}}^{L 1}\right)^{2}$, so that $\left|\eta_{\mathbf{q}}^{L n}\right| \gg 1$, and that the effect of scattering would become dominant, so that for late stages in the time 
evolution of the system the kinetic equation for harmonics waves could be approximated as follows:

$$
\begin{aligned}
\frac{\partial \mathcal{E}_{\mathbf{q}}^{L n}}{\partial \tau} \approx & \frac{\nu_{\mathbf{q}}^{L n}}{\eta_{\mathbf{q}}^{L n}} \mathcal{E}_{\mathbf{q}}^{L n} \propto \pi \int d \mathbf{u} \frac{z_{\mathbf{q}}^{L n}-z_{\mathbf{q}^{\prime}}^{L(n-1)}}{\left(\mathbf{q}-\mathbf{q}^{\prime}\right)^{2}}\left(\mathbf{q}-\mathbf{q}^{\prime}\right) \cdot \frac{\partial \Phi_{e}}{\partial \mathbf{u}} \\
& \times \delta\left[z_{\mathbf{q}}^{L n}-z_{\mathbf{q}^{\prime}}^{L(n-1)}-\left(\mathbf{q}-\mathbf{q}^{\prime}\right) \cdot \mathbf{u}\right] \mathcal{E}_{\mathbf{q}} .
\end{aligned}
$$

The above conjecture is one of the questions that the present paper seeks to address. In the equation for the particle distribution functions, the term with $g$ describes the effects of spontaneous fluctuations, and the term with the velocity derivative describes the quasilinear diffusion process. For more details on the derivation of the above equations, the reader is referred to Refs. 13, 12, and 11 .

\section{NUMERICAL ANALYSIS}

The objective of the present paper is to investigate the initial configurations that are such that ions are considered stationary, and electrons as well as the waves evolve in time. The ion distribution in 2D velocity space in dimensionless form is given by

$$
\Phi_{i}(\mathbf{u})=\frac{1}{\pi} \frac{T_{e}}{T_{i}} \frac{m_{i}}{m_{e}} \exp \left(-\frac{m_{i}}{m_{e}} \frac{T_{e}}{T_{i}} u^{2}\right) .
$$

The initial electron distribution function is assumed to be made of a Maxwellian background population and a forward-propagating beam component, with number density assigned by $n_{f}$. In 2D and dimensionless variables the electron distribution is given as follows:

$$
\begin{aligned}
\Phi_{e}(\mathbf{u}, 0)= & \frac{1}{\pi}\left(1-\frac{n_{f}}{n_{0}}\right) \exp \left[-u_{\perp}^{2}-\left(u_{\|}-\frac{v_{0}}{v_{t e}}\right)^{2}\right] \\
& +\frac{1}{\pi} \frac{n_{f}}{n_{0}} \frac{T_{e}}{T_{f}} \exp \left\{-\frac{T_{e}}{T_{f}}\left[u_{\perp}^{2}+\left(u_{\|}-\frac{v_{f}}{v_{t f}}\right)^{2}\right]\right\} .
\end{aligned}
$$

Here, $v_{t e}=\left(2 T_{e} / m_{e}\right)^{1 / 2}$ and $v_{t f}=\left(2 T_{f} / m_{e}\right)^{1 / 2}$ are the background and forward-beam thermal speeds, respectively, and $v_{0}, v_{f}$ are the drift velocities associated with the background and the forward beam, respectively. The drift velocity for the background $v_{0}$ is chosen in such a way that it guarantees zero net drift velocity for the total electron distribution, i.e., $v_{0}=-\left(n_{f} v_{f}\right) /\left(n_{0}-n_{f}\right)$.

The initial spectra of the normal modes are given by the following expressions, obtained from the balance between quasilinear and spontaneous effects, assuming the initial plasmas at thermodynamic equilibrium

$$
\begin{aligned}
\mathcal{E}_{\mathbf{q}}^{\sigma L}(0) & =\frac{g}{2\left(z_{\mathbf{q}}^{L}\right)^{2}}, \\
\mathcal{E}_{\mathbf{q}}^{\sigma S}(0) & =\frac{g}{2 z_{\mathbf{q}}^{L} z_{\mathbf{q}}^{S}} \frac{\exp \left(-\zeta_{\mathbf{q}}^{2}\right)+\rho^{1 / 2} \exp \left(-\rho \zeta_{\mathbf{q}}^{2}\right)}{\exp \left(-\zeta_{\mathbf{q}}^{2}\right)+\left(T_{e} / T_{i}\right) \rho^{1 / 2} \exp \left(-\rho \zeta_{\mathbf{q}}^{2}\right)}, \\
\zeta_{\mathbf{q}}^{2} & =\frac{\left(z_{\mathbf{q}}^{S}\right)^{2}}{q^{2}}, \quad \rho=\frac{m_{i}}{m_{e}} \frac{T_{e}}{T_{i}}
\end{aligned}
$$

For the harmonic waves, the initial spectra can be estimated using the following expression: ${ }^{11}$

$$
\mathcal{E}_{\mathbf{q}}^{L n}(0)=\frac{n(9 / 4+\Gamma)}{\left[\left(n^{2}-1\right) U^{2}-3 / 2\right]^{2}+\Gamma} \mathcal{E}_{\mathbf{q}}^{\sigma L}(0),
$$

where $\Gamma=4 \pi U^{10} e^{-2 U^{2}}, U=V_{0} / v_{t h}^{e}$, with $V_{0}$ being the beam velocity. For the present analysis, we will include only the effect of the harmonic $n=2$, and we will use the notation $N$ to denote the harmonic waves.

The set of Equations (1)-(3) for the waves $(L, S$, and $N)$ and Eq. (12) for the electrons are solved in 2D wave number space and 2D velocity space, by employing a splitting method with fixed time step for the evolution of the distribution and a Runge-Kutta method with the same fixed time step for the wave equations. The ion distribution is assumed to be fixed along all the time evolution of the system.

For all the numerical examples to be discussed subsequently, we use the normalized time interval $\Delta \tau=0.1$. We employ $51 \times 51$ grids for $q_{\perp}$ and $q_{\|}$, with $0<q_{\perp}=k_{\perp} v_{t e} /$ $\omega_{p}<0.6$, and $0<q_{\|}=k_{\|} v_{t e} / \omega_{p}<0.6$. For the velocities, we use a $51 \times 101$ grid for the $\left(u_{\perp}, u_{\|}\right)=\left(v_{\perp} / v_{t e}, v_{\|} / v_{t e}\right)$ space, covering the velocity range $0<u_{\perp}=v_{\perp} / v_{t e}<12$ and $-12<u_{\|}=v_{\|} / v_{t e}<12$. For subsequent numerical solutions, we assume the plasma parameter given by $\left(\hat{n} \lambda_{D}^{3}\right)^{-1}$ $=5.0 \times 10^{-3}$, and assume that the beam velocity is $v_{f} / v_{t e}=5.0$, with beam temperature $T_{f} / T_{e}=1.0$ and ratio of electron and ion temperature $T_{e} / T_{i}=7.0$.

Initially, we consider the case in which the relative density of the beam is $n_{f} / n_{e}=2.0 \times 10^{-4}$. For this case, in Figure 1 we present the normalized intensity of the spectrum of $L$ waves, as a function of the components of the normalized wavenumber, $q_{\perp}=k_{\perp} v_{t e} / \omega_{p}$ and $q_{\|}=k_{\|} v_{t e} / \omega_{p}$, in vertical logarithmic scale, taking into account only spontaneous and induced emission in Eq. (1). Figure 1(a) shows the spectrum at $\tau=500$, Figure 1(b) the case of $\tau=1000$, Figure 1(c) the case of $\tau=2000$, and Figure 1(d) the case of $\tau=4000$. The sequence of figures shows the growth of the primary peak of Langmuir waves, at $q_{\|} \simeq 0.2$, which is the position of the wave-particle resonance with the beam particles. The peak starts to grow at early time and is seen to grow between $\tau=500$ and $\tau=1000$, and appears stabilized after $\tau=1000$.

In Figure 2 we present the normalized intensity of the spectrum of $N$ waves, also as a function of the components of the normalized wavenumber, $q_{\perp}$ and $q_{\|}$, in vertical logarithmic scale. For the evolution of $N$ waves as shown in Figure 2, we have taken into account only the induced emission term, in Eq. (3). The spectrum of $N$ wave at $\tau=500$ is shown in Figure 2(a), at $\tau=1000$ in Figure 2(b), at $\tau=$ 2000 in Figure 2(c), and at $\tau=4000$ in Figure 2(d). The sequence of figures shows the fast growth of the peak corresponding to harmonic waves, at $q_{\|} \simeq 0.5$, which is the position of the wave-particle resonance between the harmonic waves of frequency given by $z_{\mathbf{q}}^{N}$ with the beam particles, and also shows the decay of the initial spectrum in the region adjacent to the peak, due to Landau damping. It is to be noted that the region with $q_{\|}<0$ remains unchanged, because we 

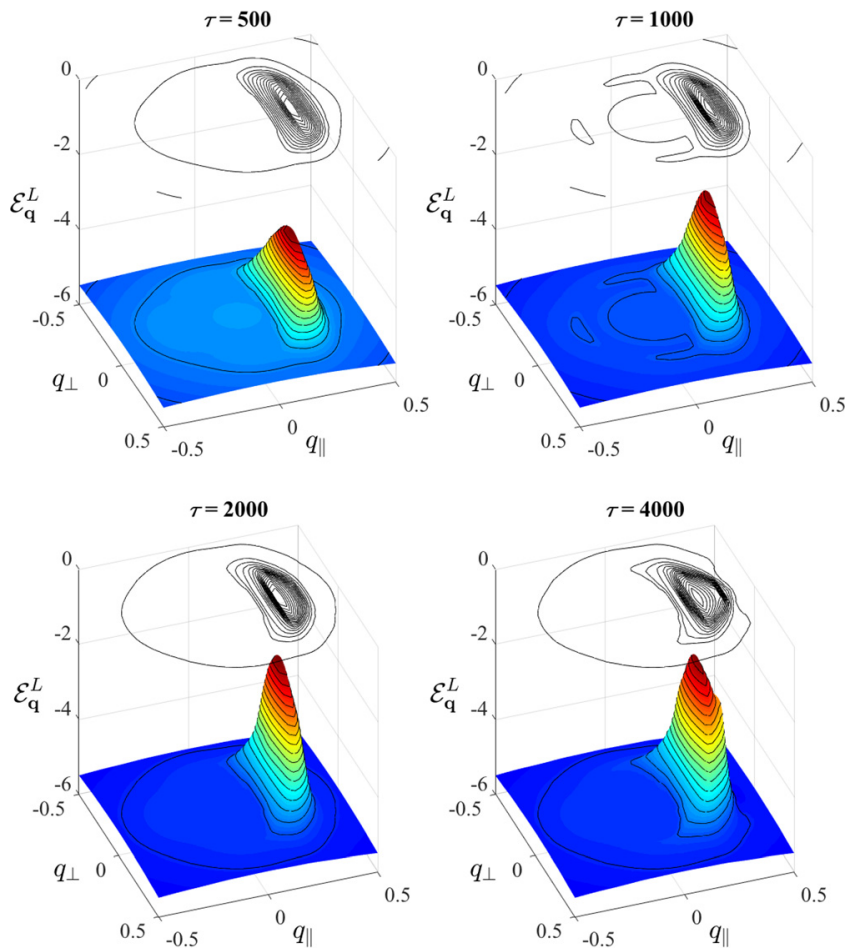

FIG. 1. Normalized L wave intensity, vs $q_{\perp}=k_{\perp} v_{t e} / \omega_{p}$ and $q_{\|}=k_{\|} v_{t e} / \omega_{p}$, in vertical logarithmic scale, taking into account only spontaneous and induced emission in the equation for $L$ wave evolution. (a) $\tau=500$; (b) $\tau=1000$; (c) $\tau=2000$; and (d) $\tau=4000$. Input parameters are $n_{f} / n_{e}$ $=2.0 \times 10^{-4}, \quad v_{f} / v_{t e}=5.0, T_{f} / T_{e}=1.0, T_{e} / T_{i}=7.0, \quad$ and $\left(\hat{n} \lambda_{D}^{3}\right)^{-1}$ $=5.0 \times 10^{-3}$.
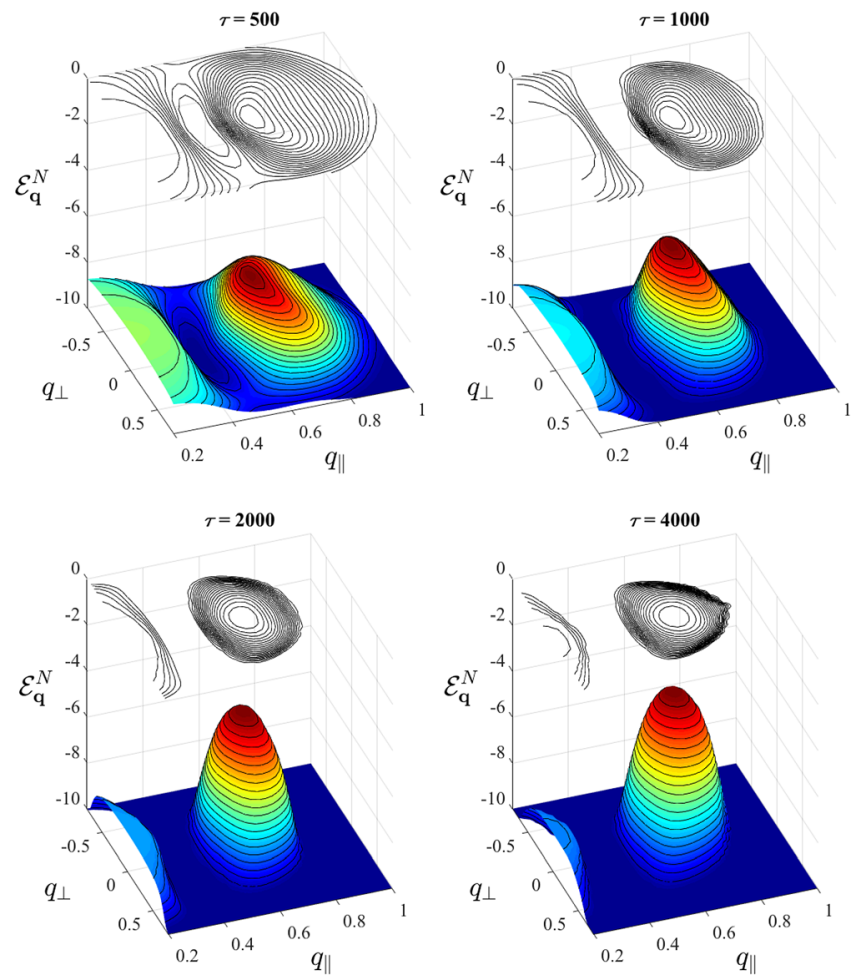

FIG. 2. Normalized $N$ wave intensity, vs $q_{\perp}=k_{\perp} v_{t e} / \omega_{p}$ and $q_{\|}=k_{\|} v_{t e} / \omega_{p}$, in vertical logarithmic scale, taking into account only induced emission in the equation for $\mathrm{N}$ wave evolution. (a) $\tau=500$; (b) $\tau=1000$; (c) $\tau=2000$; and (d) $\tau=4000$. Input parameters are the same as in Figure 1.
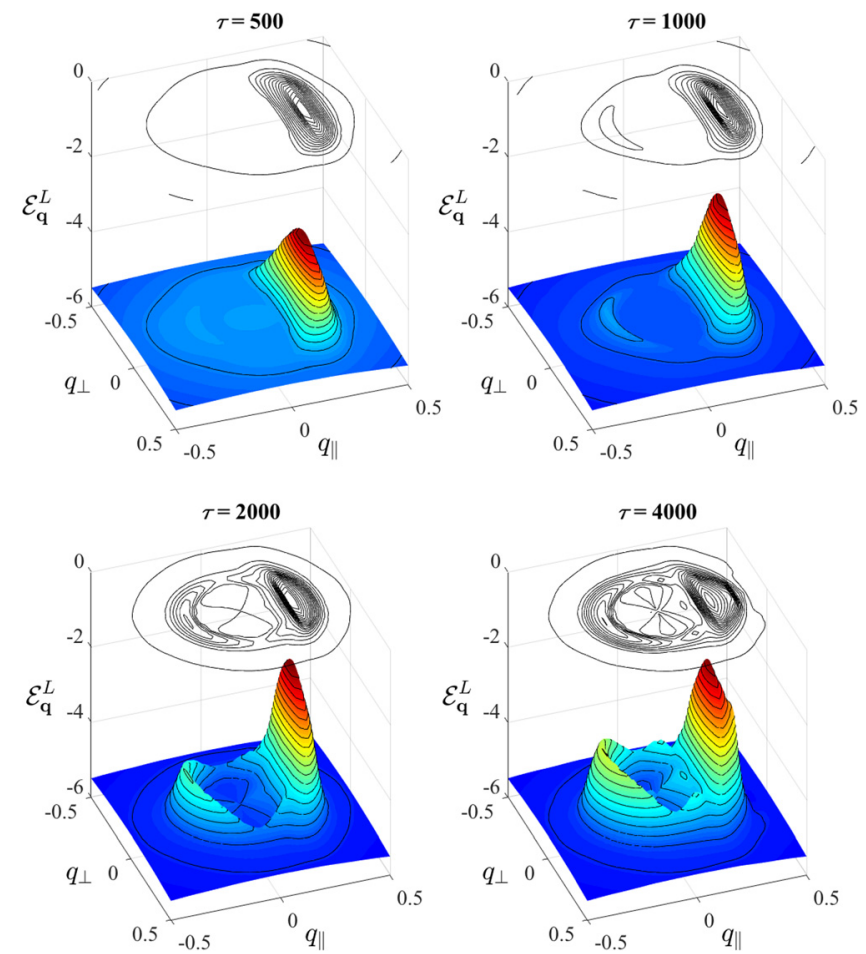

FIG. 3. Normalized $L$ wave intensity, vs $q_{\perp}=k_{\perp} v_{t e} / \omega_{p}$ and $q_{\|}=k_{\|} v_{t e} / \omega_{p}$ in vertical logarithmic scale, taking into account spontaneous and induced emission and scattering in the equation for evolution of the $L$ wave. (a) $\tau=500$; (b) $\tau=1000$; (c) $\tau=2000$; and (d) $\tau=4000$. Input parameters are the same as in Figure 1.

have not included in the theory the possibility of evolution of backward propagating harmonic waves, based on the fact that backward harmonic waves have not been seen in numerical simulations, ${ }^{28,31}$ or at least the backward harmonic waves are much more weaker in comparison with the forward $N$ waves. ${ }^{43}$ Since the $N$ mode spectra for negative $q$ do not change from their initial levels, we chose to plot the results only over positive range, $0.2<q_{\|}<1$. The peak seems to attain maximum height between $\tau=1000$ and 2000, Figures 2(b) and 2(c), while the damping continues to occur, with considerable evolution being noticeable between Figures 2(c) and 2(d).

In Figures 3 and 4 we present results which are similar to those presented in Figures 1 and 2, with the difference that in the case of Figures 3 and 4 we add the effect of the scattering to the equations for wave evolution. The results are also presented for the values of $\tau=500,1000,2000$, and 4000, corresponding to panels (a), (b), (c), and (d), respectively.

In Figure 3 we wee the evolution of $L$ waves. Panel (a) shows the spectrum at $\tau=500$, with the primary peak of $\mathrm{L}$ waves very well defined, similar to what was seen in Figure 1(a). At $\tau=1000$, Figure 3(b), one already notices the presence of backward propagating waves and formation of a ring-like structure in wavenumber space, as already reported previously, in studies that did not include the presence of harmonic waves. ${ }^{45}$ These features become more conspicuous along time evolution, and are seen fully developed at $\tau=4000$, in panel (d) of Figure 3 .

The evolution of the harmonic waves in the presence of induced emission and scattering is seen in Figure 4. The four 

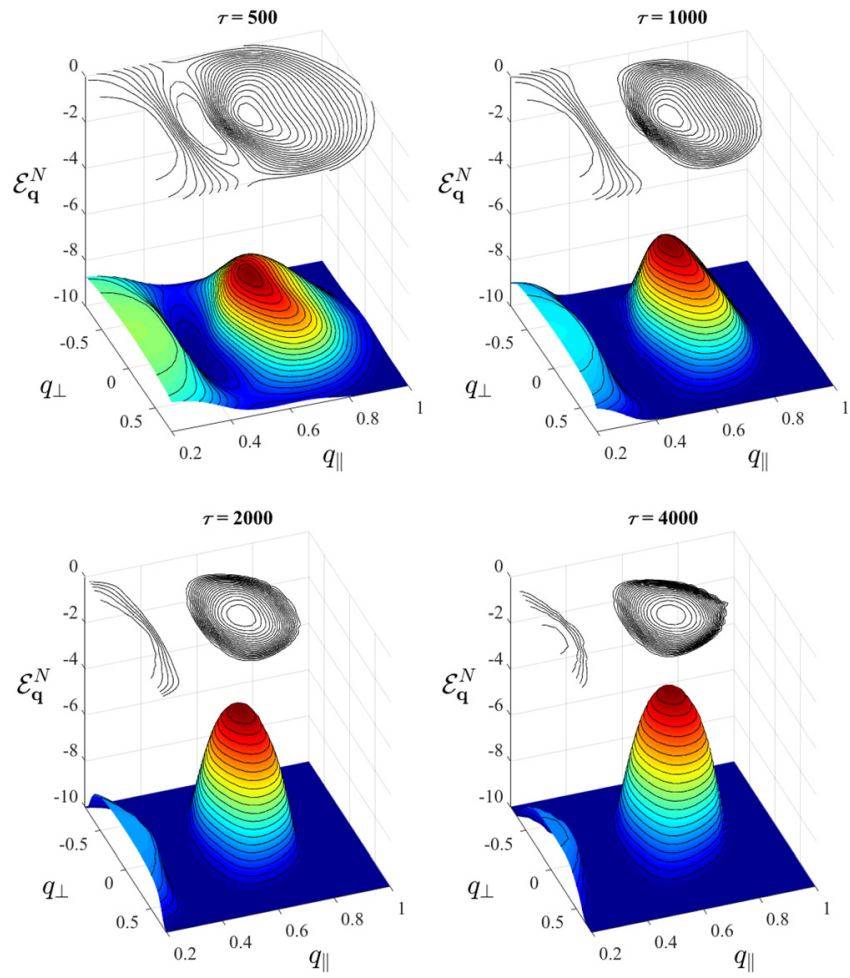

FIG. 4. Normalized $N$ wave intensity, vs $q_{\perp}=k_{\perp} v_{t e} / \omega_{p}$ and $q_{\|}=k_{\|} v_{t e} / \omega_{p}$, in vertical logarithmic scale, taking into account spontaneous and induced emission and scattering in the equation for evolution of the $N$ wave. (a) $\tau=500$; (b) $\tau=1000$; (c) $\tau=2000$; and (d) $\tau=4000$. Input parameters are the same as in Figure 1.

panels of Figure 4 are very similar to the corresponding panels of Figure 2, indicating that, for the case considered, the scattering has not produced noticeable effects in the evolution of $N$ waves, even after a time interval, from 0 to 4000 , in which significant effects due to scattering have been seen in the spectrum of $L$ waves.

In Figure 5 we show results obtained in a case with higher beam density, by considering $n_{f} / n_{e}=6.0 \times 10^{-4}$. In Figure 5 top panel we show the spectrum of $L$ waves at $\tau=4000$, obtained by taking into account only spontaneous and induced emission processes in Eq. (1). Despite the fact that the time evolution of the primary peak of $L$ waves is faster in the case of higher beam density (not shown), the comparison between Figure 5 (top) and Figure 1(d) shows that the peaks obtained at $\tau=4000$ have similar heights. The difference is that the peak is somewhat wider in the case of higher beam density. The spectrum of $L$ waves obtained at $\tau=4000$, when scattering effects are also taken into account in the evolution equation, is seen in Figure 5 (bottom panel). The spectrum obtained is similar to that obtained in the case of lower density beam, Figure 3(d), but in addition to wider forward peak, the case of higher beam density features more pronounced peak of backward waves and more pronounced ring-like feature in wavenumber space.

The corresponding spectra obtained for $N$ waves are superposed in Figures 5 (top), without scattering, and 5 (bottom), with scattering taken into account. As already noticed in the case of lower density beam, the presence of scattering has not produced significant effects on the time evolution of the harmonic waves. Note that Fig. 5 (bottom) compares
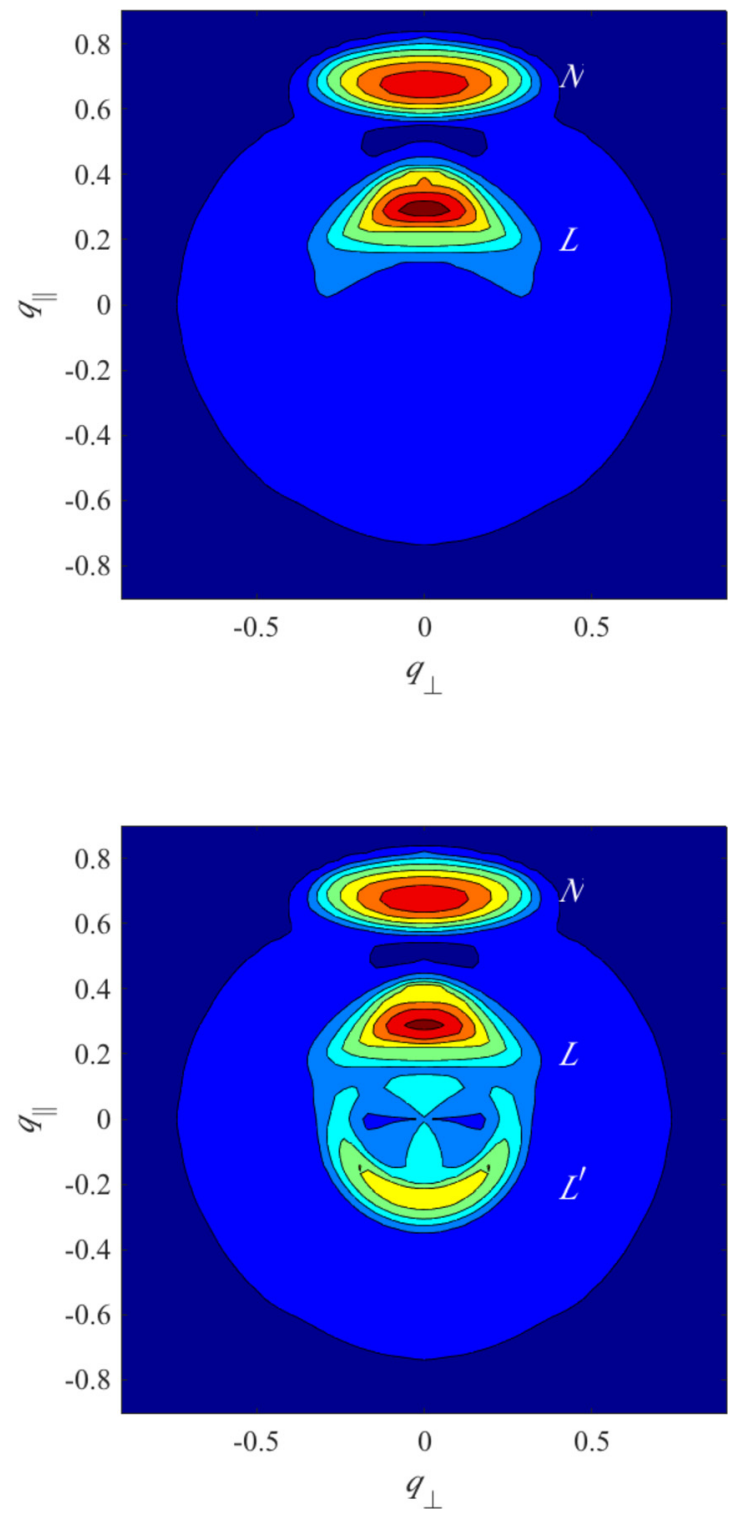

FIG. 5. Contour plots for normalized wave intensities, vs $q_{\perp}=k_{\perp} v_{t e} / \omega_{p}$ and $q_{\|}=k_{\|} v_{t e} / \omega_{p}$, at $\tau=4000$. The beam density is such that $n_{f} / n_{e}=6.0 \times 10^{-4}$, and other parameters are as in Figure 1. (Top) $L$ wave, obtained taking into account only spontaneous and induced emission in the equation for $L$ wave evolution, and $N$ wave, obtained taking into account only the effect of induced emission in the equation for $N$ wave evolution. (Bottom) $L$ wave, obtained taking into account spontaneous and induced emission and wave scattering in the equation for $L$ wave evolution, and $N$ wave, obtained taking into account induced emission and wave scattering in the equation for $N$ wave evolution.

quite reasonably with Figures 3(b) and 3(c) of Ref. 43, which show the $2 \mathrm{D}$ spectrum of fundamental and second harmonic electric field.

In Figure 6 we show the spectra of $L$ and $N$ waves as a function of absolute value of the normalized wave number, $q$, obtained after integration over the pitch-angle in wavenumber space. In all the four panels of Figure 6 one finds plotted curves corresponding to $\tau=100,200,500,1000$, $2000,3000,4000,5000$, and 6000 . The curve for $\tau=100$ is denoted in green, the curve for $\tau=1000$ is blue, the case of $\tau=3000$ is depicted using magenta color, and $\tau=6000$ appears in red. The other lines corresponding to $\tau=200$, $500,2000,4000$, and 5000, are denoted in black. 

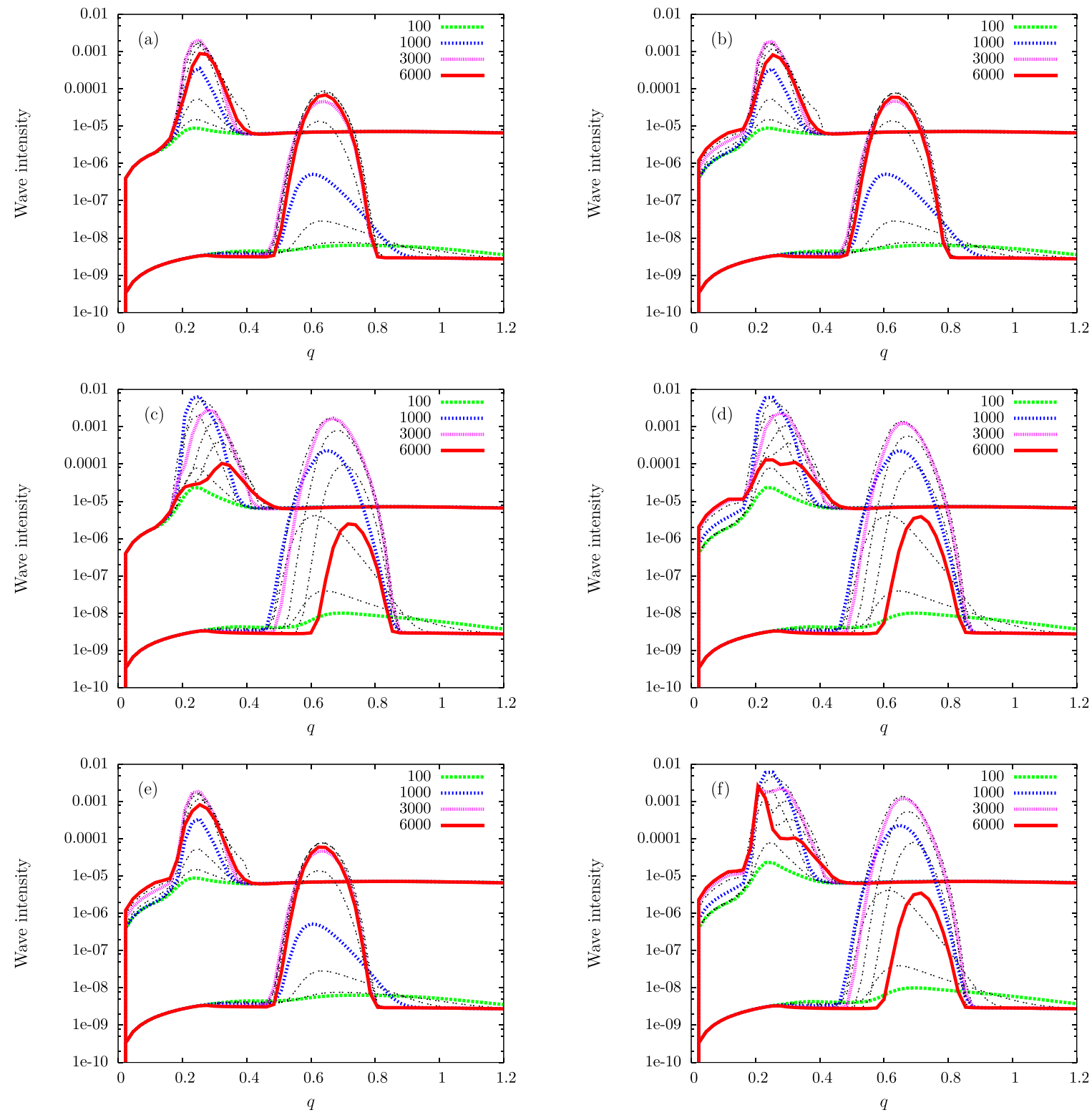

FIG. 6. Normalized $L$ and $N$ wave intensities vs. $q$, in vertical logarithmic scale, for several values of $\tau(100,200,500,1000,2000,3000,4000,5000$, and 6000). Green lines: $\tau=100$; blue lines: $\tau=1000$; magenta lines: $\tau=3000$; and red lines: $\tau=6000$. The results corresponding to 200, 500, 2000, 4000, and 5000 are depicted by black lines. Except where explicitly indicated, the parameters are the same as in Figure 1. (a) Obtained taking into account only spontaneous and induced emission in the equation for $L$ waves and induced emission in the equation for $N$ waves. (b) Spontaneous and induced emission and wave scattering in the equation for $L$ waves, and induced emission and scattering in the equation for $N$ waves. (c) Spontaneous and induced emission in the equation for $L$ waves and induced emission in the equation for $N$ waves, with $n_{f} / n_{0}=6.0 \times 10^{-4}$. (d) Spontaneous and induced emission and wave scattering in the equation for $L$ waves, and induced emission and scattering in the equation for $N$ waves, with $n_{f} / n_{0}=6.0 \times 10^{-4}$. (e) Spontaneous and induced emission, wave scattering, and three-wave decay, in the equation for $L$ waves, and induced emission and scattering in the equation for $N$ waves, with $n_{f} / n_{0}=2.0 \times 10^{-4}$. (f) Spontaneous and induced emission, wave scattering, and three-wave decay, in the equation for $L$ waves, and induced emission and scattering in the equation for $N$ waves, with $n_{f} / n_{0}=6.0 \times 10^{-4}$.

Figure 6(a) depicts results obtained for the case of $n_{f} / n_{e}=2.0 \times 10^{-4}$, by taking into account only spontaneous and induced emission processes in Eq. (1), and only induced processes in Eq. (3). The peak in the spectrum of $L$ waves starts to grow in the region slightly above $q=0.2$, grows regularly until attaining maximum value near time $\tau=3000$, and then starts a slow decrease, while undergoing a slight broadening process, as seen in Figure 1. The peak of $N$ waves appears as broad perturbation for $\tau=100$, but then grows around the value $q \simeq 0.65$, attaining maximum value near $\tau=4,000$, and then starting to decrease very slowly. The maximum height attained by the peak in $N$ waves is seen to be about $1 / 20$ of the maximum of the $L$ wave peak.

The results obtained for $n_{f} / n_{e}=2.0 \times 10^{-4}$ when scattering effects are also taken into account are seen in Fig. 6(b). The results obtained are very similar to those obtained when the scattering effects were neglected, appearing in panel (a). Particularly, the spectra of $N$ waves can hardly be distinguished, in the scale of the figure. In the case of $L$ waves, the ring-like structure formed in wavenumber space by effect of scattering appears as an enhancement of the 
spectrum in the region of $q \leq 0.2$, seen in Figure 6(b) and not seen in Figure 6(a).

In Figures 6(c) and 6(d) we show results obtained considering the case of higher beam density, $n_{f} / n_{e}=6.0$ $\times 10^{-4}$. The results appearing in Figure 6(c) were obtained without taking into account scattering effects in the time evolution of the waves, while those in Figure 6(d) were obtained with scattering effects taken into account. The comparison between the results appearing in panels (c) and (d) and those appearing in panels (a) and (b) show that the time evolution is faster in the case of higher density beam, so that the late evolution on the system accumulates considerable difference between the two cases. Figure 6(c) shows that the maximum in the $L$ spectrum is attained for $\tau$ near the value $\tau=1000$, and that the peak in the spectrum decreases considerably afterward, up the final time of $\tau=6000$ which has been shown in the figure.

The comparison between Figures 6(c) and 6(d) show that the presence of scattering caused considerable distortion in the wave spectrum at later times, in addition to the presence of the ring-like structure which enhances the spectrum in the region of small wavenumber. On the other hand, Figures 6(c) and 6(d) show that the growth of $N$ waves is such that they attain maximum height for $\tau$ between 2000 and 3000 , before decreasing. It is interesting to point out that, for $\tau \leq 1000$, the curves obtained for $N$ waves without taking into account the scattering and with scattering are basically the same. For $\tau$ in the range $2000 \leq \tau \leq 5000$, it is seen from panels (c) and (d) of Figure 6 that the peak of $N$ waves obtained with scattering is slightly lower than the peak obtained without including the effect of scattering. However, for $\tau=6000$, it is seen that the peak obtained with scattering, in Figure 6(d), is somewhat higher than the peak appearing in Figure 6(c), obtained without scattering. This feature has to be more carefully investigated, in order to see if it is due to a real physical process, or if it is due to numerical instability appearing at late stages of the time evolution.

Figure 6 features two other panels, in which we present results obtained from the numerical solution of the system of coupled equations, including also effects of three-wave decay in the equation for evolution of $L$ and $S$ waves. That is, for the results shown in Figures 6(e) and 6(f), the evolution of $L$ waves suffers the influence of spontaneous and induced emission, three-wave decay and scattering, the evolution of $S$ waves suffers the influence of spontaneous and induced emission and three-wave decay, and the evolution of $N$ waves occurs under the influence of induced emission and scattering. Figure 6(e) shows the results obtained for $L$ and $N$ waves, for several values of normalized $\tau$, considering the case of $n_{f} / n_{e}=2.0 \times 10^{-4}$. The comparison with the results shown in Figure 6(b), obtained for the same parameters without taking into account the effect of three-wave decay for $L$ and $S$ waves, shows that the effect of the three-wave decay term is barely noticeable in the integrated spectra of $L$ waves. In Figure 6(f) we show similar results, obtained for the case $n_{f} / n_{e}=6.0 \times 10^{-4}$. The comparison with Figure 6(d), obtained for the same parameters, shows that the early time evolution is quite similar in both cases, but for late time it is quite noticeable in Figure 6(f) the enhancement of the $L$ spectrum which occurs in the case of addition of three-wave decay and scattering effects.

Finally, in Figure 7 we present some results concerning the time evolution of the coefficients associated to the time evolution of the harmonic waves. We present these coefficients as a function of the absolute value of the normalized wavenumber, after integration over the wave vector pitch angle. The coefficient associated to wave damping or growth by quasilinear processes will be denominated as $\gamma_{q}^{N}$, the coefficient associated to scattering is $\nu_{q}^{N}$, and the coefficient which appears at the denominator of both the quasilinear and the scattering term in Eq. (3) will be denoted by $\eta_{q}^{N}$. In Fig. 7 these coefficients are depicted for several values of the normalized time, $\tau=100,200,500,1000,2000,3000,4000$, 5000 , and 6000 . We consider two cases of the beam relative density, with other parameters as in Fig. 1.

Figure 7(a) shows $\gamma_{q}^{N}$ vs. $q$, for the case $n_{f} / n_{e}=2.0 \times 10^{-4}$. The case of $\tau=100$ is representative of the early time of wave evolution. It is seen that for small $q$ there is negligible damping, due to resonance with the very small population at the tail of the distribution. For $0.3 \leq q \leq$ 0.6 there is more significant damping, at the far extreme of the beam, and for $0.6 \leq q \leq 0.9$ the positive value of $\gamma_{q}^{N}$ indicates wave growth. For larger values of $q$, the damping becomes again very significant, due to resonance with particles of the Maxwellian part of the distribution. Figure 7(a) shows that along the time evolution the peak of wave growth is displaced toward smaller values of $q$. Between $\tau=500$ and $\tau=1000$ the wave growth starts to decrease, as indicated by the lines blue and magenta, respectively. This is due to formation of a plateau in the distribution function. Shortly after that, the peak of harmonic starts to be reabsorbed by the particles, as indicated by the negative values of $\gamma$ for all values of $q$, in the curves depicting the situation at $\tau=2000$, and beyond. This is because the flat plateau is seen in the 1D projection of the distribution function, along parallel direction. In 2D space, there is always regions of negative derivatives around the position of the beam in velocity space, which dominate the evolution after the positive derivatives in the region of the beam are flattened out.

In Figure 7(b) we have information about the time evolution of $\gamma_{q}^{N}$ vs. $q$, in the case of higher beam density, $n f / n_{e}=6.0 \times 10^{-4}$. It is seen that qualitatively the results are similar to those appearing in Figure 7(a), except that the maximum growth or damping rates are larger, and the time evolution is faster. The maximum growth coefficient is seen to occur for $\tau \simeq 200$ in Figure 7(b), with significant reduction of the growth already seen in the case of $\tau=500$, as depicted by the blue line.

The evolution of $\nu_{q}^{N}$, the coefficient associated to scattering, is seen in Figures 7(c) and 7(d). It is seen that the scattering coefficient is negative for all values of $q$, indicating that the scattering effect contributes to deplete the peak of harmonic waves, spreading the wave intensity. However, it is seen that the effect is weak, as compared with the quasilinear effect $\left(\left|\eta_{q}^{N} / \gamma_{q}^{L}\right| \sim 10^{-2}\right)$. In the case of lower beam density, in Figure 7(c), it is seen that the maximum absolute value of $\gamma_{a}^{N}$ is attained for $\tau \simeq 3000$, approximately the time of the maximum of the spectrum of $N$ waves, and that this 

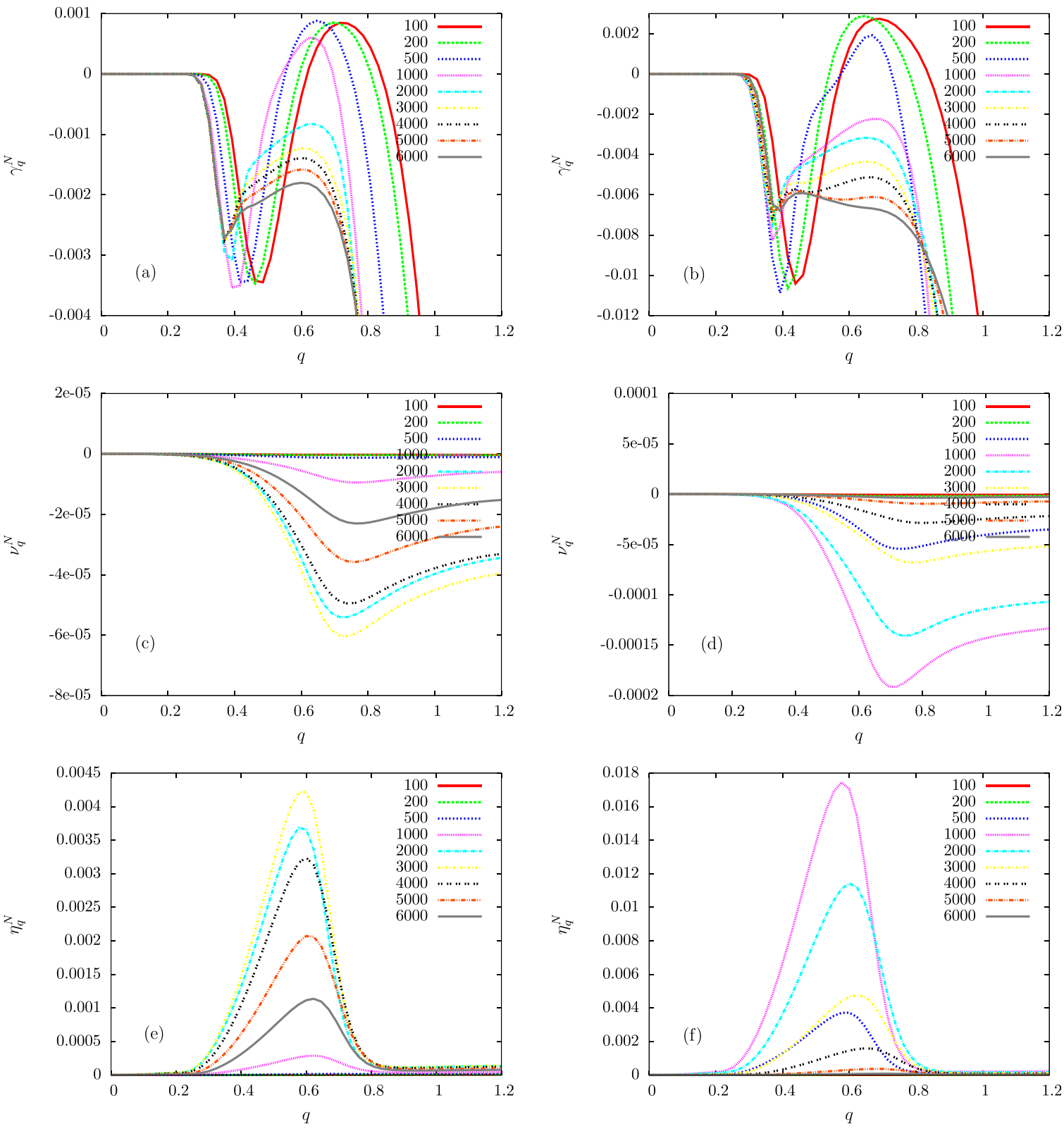

FIG. 7. Normalized coefficients associated to the time evolution of harmonic waves, as described by Eq. (3), vs. normalized wavenumber, for several values of $\tau$ $\left(100,200,500,1000,2000,3000,4000,5000\right.$, and 6000). (a) $\gamma_{q}^{N}$ vs. q, $n_{f} / n_{e}=2.0 \times 10^{-4}$; (b) $\gamma_{q}^{N}$ vs. q, $n_{f} / n_{e}=6.0 \times 10^{-4}$; (c) $\nu_{q}^{N}$ vs. q, $n_{f} / n_{e}=2.0 \times 10^{-4}$; (d) $\nu_{q}^{N}$ vs. q, $n_{f} / n_{e}=6.0 \times 10^{-4}$; (e) $\eta_{q}^{N}$ vs. q, $n_{f} / n_{e}=2.0 \times 10^{-4}$; and (f) $\eta_{q}^{N}$ vs. q, $n_{f} / n_{e}=6.0 \times 10^{-4}$. Other parameters are as in Figure 1 .

maximum absolute value is smaller than the maximum absolute value of $\gamma_{q}^{N}$ at corresponding time. Similar comments can be made about the results obtained in the case of higher density beam, as depicted in Fig. 7(d).

The values of the coefficient $\eta_{q}^{N}$, vs. $q$, for several values of $\tau$, appear in Figures 7(e) and 7(f). For the case of $n_{f} / n_{e}=2.0 \times 10^{-4}$, seen in Figure $7(\mathrm{e})$, it is seen that the quantity $\eta$ features a peak which coincides with the location of the peak of $N$ waves in wavenumber space, and that the maximum value is attained for $\tau \simeq 3000$, about the time of the maximum value of the wave spectra. For $\tau \leq 500$, it is seen that the absolute value of $\eta_{q}^{N}$ is indeed very small, which, together with the corresponding small value of $\left|\nu_{q}^{N}\right|$, confirms the conjecture presented in Eq. (17). However, Figure 7(e) shows that the maximum value of $\eta_{q}^{N}$ never surpasses the value $4.5 \times 10^{-3}$, which means that the numerical analysis made in two dimensions has not confirmed the conjecture made in Ref. 11 and presented in Eq. (18). Comments which are qualitatively similar can be made for higher beam density, except that the evolution is faster and the maximum attained by $\gamma_{q}^{N}$ is larger, being about $1.8 \times 10^{-2}$ in the case of $n_{f} / n_{e}=6.0 \times 10^{-4}$, shown in Figure 7(f).

Hence, we conclude that the main saturation mechanism for the nonlinear eigenmode is the quasilinear process of plateau formation in the electron distribution function, with the consequent arrest of the weak-beam instability.

\section{SUMMARY}

In the present paper we have presented results of numerical analysis of the generalized weak turbulence theory in two dimensional space, taking into account the normal 
electrostatic modes, which are Langmuir and ion-acoustic waves, and also the occurrence of harmonic waves with frequency which is about twice the frequency of fundamental Langmuir waves. The wave equations were solved along with the equation for time evolution of the electron distribution function, providing what is possibly the first examples of self-consistent evolution of electrostatic waves, including the harmonic of Langmuir waves, in more than one dimension. The equation which has been derived for the evolution of harmonic waves contains the effect which is known as induced emission and the nonlinear effect which is denominated as scattering, or nonlinear Landau damping. We have also taken into account the mechanisms of scattering and induced emission, along with the spontaneous emission effect, in the equation for the evolution of fundamental Langmuir waves, and the spontaneous and induced emission effects in the equation for ion-acoustic waves, where the scattering effect is regarded as negligible.

Reference 43 contains a Figure that shows the 2D spectrum of $L$ and $N$ modes, where while $L$ mode and $N$ mode spectra are similar in overall shape, $N$ mode spectra occupy a broader range of $k$ space. Such a feature has not been theoretically explained. The results obtained show the growth of a peak of harmonic electrostatic waves, with width in wavenumber which is comparable with the width of the primary peak of Langmuir waves. Such a 2D spectral feature cannot be discussed in the one-dimensional approximation, and is consistent with results obtained with direct numerical simulation result reported in Ref. 43.

The main purpose of the present investigation had been to test the hypothesis put forth in Ref. 11, in which it was speculated that early dynamical evolution of $N$ mode should follow quasilinear theory, but for later times, nonlinear scattering should dominate. According to the present finding, the effect of scattering has been seen to be very small in the case of harmonic waves, whose evolution is dominated by the induced emission effect. This finding contradicts the speculation in Ref. 11.

\section{ACKNOWLEDGMENTS}

E.C.F.-P. acknowledges support from CNPq (Brazil). L.F.Z. acknowledges support from CNPq (Brazil), Grant No. 304363/2014-6. R.G. acknowledges support from CNPq (Brazil), Grant Nos. 304461/2012-1 and 478728/2012-3. P.H.Y. acknowledges support by NSF Grant No. AGS1550566 to the University of Maryland. The research at Kyung Hee University was supported by the BK21-Plus grant from the National Research Foundation (NRF), Korea. P.H.Y. also acknowledges the Science Award Grant from the GFT Foundation to the University of Maryland. Part of this work was carried out while P.H.Y. was visiting Ruhr University Bochum, Germany, which was made possible by support from the Ruhr University Research School PLUS, funded by Germany's Excellence Initiative (DFG GSC 98/3).

\footnotetext{
${ }^{1}$ B. B. Kadomtsev, Plasma Turbulence (Academic Press, New York, 1965). ${ }^{2}$ R. Z. Sagdeev and A. A. Galeev, Nonlinear Plasma Theory (Benjamin, New York, 1969).
}

${ }^{3}$ V. N. Tsytovich, Nonlinear Effects in a Plasma (Plenum, New York, 1970).

${ }^{4}$ R. C. Davidson, Methods in Nonlinear Plasma Theory (Academic Press, New York, 1972).

${ }^{5}$ A. I. Akhiezer, I. A. Akhiezer, R. V. Polovin, A. G. Sitenko, and K. N. Stepanov, Plasma Electrodynamics: Non-Linear Theory and Fluctuations (Pergamon, New York, 1975), Vol. 2.

${ }^{6} \mathrm{~V}$. N. Tsytovich, An Introduction to the Theory of Plasma Turbulence (Pergamon, New York, 1977).

${ }^{7}$ D. B. Melrose, Plasma Astrophysics (Gordon and Breach, New York, 1980).

${ }^{8}$ A. G. Sitenko, Fluctuations and Nonlinear Wave Interactions in Plasmas (Pergamon, New York, 1982).

${ }^{9}$ V. N. Tsytovich, Lectures on Nonlinear Plasma Kinetics (Springer, New York, 1995).

${ }^{10}$ P. H. Yoon, Phys. Plasmas 7, 4858 (2000).

${ }^{11}$ P. H. Yoon, Phys. Plasmas 12, 042306 (2005).

${ }^{12}$ P. H. Yoon, Phys. Plasmas 13, 022302 (2006).

${ }^{13}$ P. H. Yoon, L. F. Ziebell, R. Gaelzer, and J. Pavan, Phys. Plasmas 19, 102303 (2012).

${ }^{14}$ P. H. Yoon, L. F. Ziebell, E. P. Kontar, and R. Schlickeiser, Phys. Rev. E 93, 033203 (2016)

${ }^{15}$ S. F. Tigik, L. F. Ziebell, P. H. Yoon, and E. P. Kontar, Astron. Astrophys. 586, A19 (2016)

${ }^{16}$ P. H. Yoon, R. Gaelzer, T. Umeda, Y. Omura, and H. Matsumoto, Phys. Plasmas 10, 364 (2003).

${ }^{17}$ R. Gaelzer, P. H. Yoon, T. Umeda, Y. Omura, and H. Matsumoto, Phys. Plasmas 10, 373 (2003).

${ }^{18}$ T. Umeda, Y. Omura, P. H. Yoon, R. Gaelzer, and H. Matsumoto, Phys. Plasmas 10, 382 (2003).

${ }^{19}$ J. R. Apel, Phys. Rev. Lett. 19, 744 (1967)

${ }^{20}$ J. R. Apel, Phys. Fluids 12, 640 (1969).

${ }^{21}$ J. H. Malmberg and C. B. Wharton, Phys. Fluids 12, 2600 (1969).

${ }^{22}$ K. Mizuno and S. Tanaka, Phys. Rev. Lett. 29, 45 (1972).

${ }^{23}$ H. Mori, J. Phys. Soc. Jpn. 35, 592 (1973).

${ }^{24}$ P. J. Kellogg, S. J. Monson, W. Bernstein, and B. A. Whalen, J. Geophys. Res. 91, 12065, doi:10.1029/JA091iA11p12065 (1986).

${ }^{25}$ A. J. Klimas, J. Geophys. Res. 88, 9081, doi:10.1029/JA088iA11p09081 (1983).

${ }^{26}$ A. J. Klimas, J. Geophys. Res. 95, 14905, doi:10.1029/JA095iA09p14905 (1990).

${ }^{27}$ D. Schriver, M. Ashour-Abdalla, V. Sotnikov, P. Hellinger, V. Fiala, R. Bingham, and A. Mangeney, J. Geophys. Res. 105, 12919, doi:10.1029/ 1999JA000301 (2000).

${ }^{28}$ Y. Kasaba, H. Matsumoto, and Y. Omura, J. Geophys. Res. 106, 18693 doi:10.1029/2000JA000329 (2001).

${ }^{29}$ P. Travnicek, P. Hellinger, D. Schriver, and M. G. G. Taylor, Nonlinear Processes Geophys. 10, 345 (2003).

${ }^{30}$ T. Rhee, M. Woo, and C.-M. Ryu, J. Korean Phys. Soc. 54, 313 (2009).

${ }^{31}$ F. J. R. Simões, Jr., M. V. Alves, and R. Gaelzer, J. Geophys. Res. 115, A06105 (2010).

${ }^{32}$ C. Lacombe, A. Mangeney, C. H. Harvey, and J. D. Scudder, J. Geophys. Res. 90, 73, doi:10.1029/JA090iA01p00073 (1985).

${ }^{33}$ I. H. Cairns, J. Geophys. Res. 91, 2975, doi:10.1029/JA091iA03p02975 (1986).

${ }^{34}$ D. M. Malaspina, I. H. Cairns, and R. E. Ergun, J. Geophys. Res. 115, A01101 (2010).

${ }^{35}$ D. M. Malaspina, D. B. Graham, R. E. Ergun, and I. H. Cairns, J. Geophys. Res. 118, 6880, doi:10.1002/2013JA019309 (2013).

${ }^{36}$ D. B. Graham, I. H. Cairns, and D. M. Malaspina, J. Geophys. Res. 119, 723, doi:10.1002/2013JA019317 (2014).

${ }^{37}$ Y. Nariyuki and T. Umeda, Phys. Plasmas 17, 054506 (2010).

${ }^{38}$ M. A. Diaz, M. Zettergren, J. L. Semeter, and M. Oppenheim, Ann. Geophys. 30, 1169 (2012).

${ }^{39}$ J. O. Thurgood and D. Tsiklauri, Astron. Astrophys. 584, A83 (2015).

${ }^{40}$ P. H. Yoon, S. Yi, and C.-M. Ryu, Phys. Plasmas 12, 052305 (2005).

${ }^{41}$ R. Gaelzer, L. F. Ziebell, and P. H. Yoon, Phys. Plasmas 9, 96 (2002).

${ }^{42}$ P. H. Yoon, Phys. Plasmas 12, 052313 (2005).

${ }^{43}$ T. Rhee, C.-M. Ryu, M. Woo, H. H. Kaang, S. Yi, and P. H. Yoon, Astrophys. J. 694, 618 (2009).

${ }^{44}$ L. F. Ziebell, R. Gaelzer, and P. H. Yoon, Phys. Plasmas 15, 032303 (2008). 
${ }^{45}$ L. F. Ziebell, R. Gaelzer, J. Pavan, and P. H. Yoon, Plasma Phys. Controlled Fusion 50, 085011 (2008).

${ }^{46}$ L. F. Ziebell, P. H. Yoon, L. T. Petruzzellis, R. Gaelzer, and J. Pavan, Astrophys. J. 806, 237 (2015).

${ }^{47}$ L. F. Ziebell, L. T. Petruzzellis, P. H. Yoon, R. Gaelzer, and J. Pavan, Astrophys. J. 818, 61 (2016)
${ }^{48}$ L. F. Ziebell, P. H. Yoon, R. Gaelzer, and J. Pavan, Phys. Plasmas 21, 012306 (2014).

${ }^{49}$ L. F. Ziebell, P. H. Yoon, R. Gaelzer, and J. Pavan, Astrophys. J. Lett. 795, L32 (2014)

${ }^{50}$ L. F. Ziebell, P. H. Yoon, R. Gaelzer, and J. Pavan, Plasma Phys. Controlled Fusion 54, 055012 (2012). 